\title{
Distant Speech Separation using Predicted Time-Frequency Masks from Spatial Features
}

\author{
Pasi Pertilä*, Joonas Nikunen \\ Tampere University of Technology, Department of Signal Processing, P.O. Box 553, 33101-FI, Finland
}

\begin{abstract}
Speech separation algorithms are faced with a difficult task of producing high degree of separation without containing unwanted artifacts. The time-frequency (T-F) masking technique applies a real-valued (or binary) mask on top of the signal's spectrum to filter out unwanted components. The practical difficulty lies in the mask estimation. Often, using efficient masks engineered for separation performance leads to presence of unwanted musical noise artifacts in the separated signal. This lowers the perceptual quality and intelligibility of the output.

Microphone arrays have been long studied for processing of distant speech. This work uses a feed-forward neural network for mapping microphone array's spatial features into a T-F mask. Wiener filter is used as a desired mask for training the neural network using speech examples in simulated setting. The T-F masks predicted by the neural network are combined to obtain an enhanced separation mask that exploits the information regarding interference between all sources. The final mask is applied to the delay-and-sum beamformer (DSB) output.

The algorithm's objective separation capability in conjunction with the separated speech intelligibility is tested with recorded speech from distant talkers in two rooms from two distances. The results show improvement in instrumental measure for intelligibility and frequency-weighted SNR over complex-valued non-negative matrix factorization (CNMF) source separation approach, spatial sound source separation, and conventional beamforming methods such as the DSB and minimum variance distortionless response (MVDR).
\end{abstract}

Keywords: Speech separation, microphone arrays, neural networks, beamforming, time-frequency masking.

\section{Introduction}

Source separation refers to a process of estimating individual sound sources from an observed mixture of sources. In the distant talker scenario, investigated in this work, multiple speech sources are away from the array and the captured mixture contains reverberated target signals embedded in noise, which makes the separation task nontrivial. The applications of speech separation include automatic speech recognition (ASR), hearing aids, teleconferencing and post-processing of recordings.

Speech can be approximated as a sparse signal, i.e. that simultaneous speech consist of non-overlapping T-F points of each speaker. The assumption is also known as the wdisjoint orthogonality (Ylmaz and Rickard, 2004). Given a stimulus, auditory masking (Fastl and Zwicker, 1990) can cause inaudibility of lower amplitude frequencies inside a critical band. Auditory masking occurs also after the end of a stimulus, and to some degree even before the onset. Therefore, even non-overlapping interference can reduce the perception of the target signal.

\footnotetext{
* Corresponding author

Email addresses: pasi.pertila@tut.fi (Pasi Pertilä), joonas.nikunen@tut.fi (Joonas Nikunen)
}

Four main approaches for source separation can be identified (Hummersone et al., 2014): blind source separation (BSS) methods, computational audio scene analysis (CASA) approaches, spatial filtering methods such as beamforming, and the non-negative matrix factorization (NMF) approaches.

The independent component analysis (ICA) Hyvärinen and Oja, 2000) is a popular BSS scheme that can be used to separate independent and non-Gaussian source signals. ICA can be applied in the frequency domain to deal with reverberation, but since each frequency is processed separately the source permutations needs to be solved in addition (Smaragdis, 1998; Sawada et al., 2004).

T-F separation methods apply a mask on top of the observed spectrogram to separate the desired (speech) signal from interference. The ideal binary mask (IBM) assigns each T-F point a value of 1 or 0 depending whether or not the point belongs to target, and it can be considered as the goal of CASA (Wang, 2005). The real-valued ideal ratio mask (IRM), presented in Srinivasan et al. (2006), contains values in the range $[0,1]$. The well known Wiener filter (WF) can be considered as a T-F mask and it is an important special case of the IRM since it minimizes the estimation error of reconstruction. Recently, 
Hummersone et al. (2014) argued that IRM may be more closely related to auditory processes than IBM and reviewed studies favoring IRM over IBM in certain ASR tasks and speech intelligibility (SI) measurements. In a listening experiment performed by Madhu et al. (2013) the WF based mask achieved higher SI in noisy conditions over the IBM. For hearing impaired listeners with cochlear implants the WF based mask and IBM resulted in equal SI (Koning et al., 2014). The normal hearing listeners preferred the use of WF based mask over IBM in a pairwise comparison test.

Largely the difficulty in T-F based masking is the actual mask estimation. Machine learning techniques have been researched in speech enhancement and separation intensively for mono signals. Kim et al. (2009) propose a Bayesian classifier to predict the IBM using the Gaussian mixture model (GMM), trained in three types of noise. The algorithm provided SI improvement for normal hearing listeners in noise conditions of $-5 \mathrm{~dB}$, and $0 \mathrm{~dB}$. Healy et al. (2013) utilize DNNs to predict IBM, and demonstrate that the method can significantly improve SI for hearing impaired listeners. Weninger et al. (2014) used a long short-term memory (LSTM) recurrent neural network (RNN) to predict a T-F mask for speech enhancement. Narayanan and Wang (2013) use spectral features such as Mel-frequency cepstral coefficients and their delta components as the features of a DNN for obtaining an IRM. Furthermore, using NMF on the DNN output has been proposed for enhancing the perceptual quality of separated speech by Williamson et al. (2014). A combination of DNNs and support vector machines (SVMs) for speech enhancement using binary classification of T-F bands is proposed by Wang and Wang (2013). Deep recurrent autoencoder neural network is used to denoise input features in noise robust automatic speech recognition (ASR) by Maas et al. (2012). Swietojanski et al. (2013) reported that using a DNN in distant speech recognition by concatenating the amplitude based feature values from multiple channels resulted in increased speech recognition results over using the DNN with features from a single channel or a beamformer output. The approach however did not consider spatial features between multiple channels.

The NMF and non-negative matrix deconvolution (NMD) have been widely applied for monaural speech enhancement (Raj et al., 2010; Mohammadiha et al., 2013) and directly for noise-robust feature extraction for ASR (Gemmeke et al., 2011; Schuller et al., 2010). The single channel speech enhancement with NMF requires training the signal model based on a prior knowledge about the noise context or speaker identity. For source separation in a blind and unforeseen scenario the multichannel and complex-valued extensions of NMF have been proposed by Ozerov and Fevotte (2010) and by Sawada et al. (2013). The CNMF based separation proposed by Sawada et al. (2013) uses the spatial cues in conjunction with spectral redundancy in estimation of the parameters of the sources, i.e. mixing and magnitude spectrogram. The reconstruction of the sources is based on multichannel Wiener filtering, which can be regarded as a MVDR beamformer with a single channel post-filter applied to its output (Simmer et al., 2001). A comparison of separation performance of CNMF and conventional frequency-domain ICA is reported by Nikunen and Virtanen (2014a). The NMF based methods in general operate off-line both in single and multichannel case.

Introducing a second microphone enables spatial cues. The human head and torso cause source position and frequency specific modifications to the received signal. Such recordings from microphones placed in the ears are referred as binaural, and the typical cues are interaural time delay (ITD) and interaural level difference (ILD). The degenerate un-mixing estimation technique (DUET) clusters each T-F point based on its cue values (Ylmaz and Rickard, 2004), and obtains a binary mask for each cluster. Supervised learning via kernel-density estimation is used to obtain binary T-F mask values by Roman et al. (2003). The IRM prediction from binaural features was proposed by Srinivasan et al. (2006), who used it for speech enhancement based ASR. The approach was compared to missing data ASR using the IBM prediction method by Roman et al. (2003). The small vocabulary task was marginally more successfully recognized with the missing data approach, while the large vocabulary recognition task was significantly more successful using the IRM. Ayllon et al. (2013) utilize the generalized discriminant analysis (GDA) to classify binaural features to predict IBM for hearing aids. The authors analyze different types of non-linear feature transforms, using varying amounts of adjacent T-F points, and propose an evolutionary quantization scheme to achieve low transmission rate to exchange feature values between the two hearing aid devices. Woodruff and Wang (2013) propose the use of spatial cues along with pitch features for voiced frames to train two sets of multilayer perceptrons for each combination of azimuth angle and frequency band to obtain T-F masks. Jiang et al. (2014) propose IBM prediction with a deep neural network (DNN) using ITD and ILD features obtained from gammatone filtered binaural signals.

Beamforming is linear filtering of microphone array signals to amplify the desired direction while simultaneously attenuating noise and interference. Fixed beamformers, such as the delay-and-sum beamformer (DSB) pre-calculate weights for each desired direction and simply apply the direction dependent weights during operation. In contrast, adaptive beamformers continuously update the weights according to changing signal and/or noise statistics. The minimum variance distortionless response (MVDR) obtains filter weights by keeping the response to look direction undistorted while minimizing the output noise. The beamformer output is typically enhanced with a (single channel) post-filter, and post-filters for different types of noise have been designed by Zelinski (1988); Simmer et al. (2001); McCowan and Bourlard 
(2003); Lefkimmiatis and Maragos (2007). The spatial post-filter can suppress also point-wise noise sources (Tashev and Acero, 2006). Seltzer et al. (2007) presented post-filtering using phase differences and spectral observations for a linear array. A log-MMSE adaptive beamformer that uses spatial post-filtering for producing a desired signal was proposed by Seltzer and Tashev (2008) to reduce musical noise artifacts. The method proposed by Pertilä (2013) utilizes multiple target tracking (MTT) and DOA likelihood ratios to obtain a T-F mask for each source. A T-F mask can be used as a post-filter.

The mentioned spatial cues are channel pairwise, which poses no issues in two channel recordings, but in general for $M$ microphones the number of pairs grows $\mathcal{O}\left(M^{2}\right)$, which can result in huge spatial cue vectors for microphone arrays. Pertilä and Nikunen (2014) performed sound source localization (SSL) and then presented a phase difference based feature vector that is independent of the number of microphone pairs. In addition, the feature vector is independent of the absolute source angle. This enables a single feed-forward neural network (NN) to be trained to predict a Wiener filter for a source in arbitrary direction. To address the perceptual design criteria, real speech examples were presented to the NN in order to learn the structure of naturally occurring WF based masks. The post-filter was applied to DSB output of a circular microphone array. Objective measurements of SI exceeded those of DSB, while high SNR values typical for spatial post-filter methods were retained for distant talkers in an office.

This work extends the speech enhancement work of Pertilä and Nikunen (2014) to source separation by introducing a post-processing stage to include information between sources into the predicted T-F masks. Secondly, the reverberated components are treated as undesired noise by the WF in order to attenuate reverberation. Both modifications decrease the leakage of interfering sources into the target speaker, thus improving the framework's separation performance. In comparison to Pertilä (2013), the current work utilizes machine learning to predict the post-filter, instead of using simply array response ratio towards the source and other directions to form the post-filter.

Single speaker sentences from simulated noisy reverberant conditions are used to train the NN, while the method is tested with mixtures of recordings of distant talkers captured with an actual microphone array in rooms with low and moderate reverberation. The algorithm first locates each source of the mixture using SSL and predicts the corresponding T-F masks. A post-processed T-F mask is then applied to the DSB output of each source in order to separate it. Only a single NN is required to separate any number of sources in any spatial configuration in all frequencies. The training signals do not need to include multiple simultaneous talkers which keeps the complexity of training low. In addition, the T-F mask prediction is a simple process of obtaining the NN output values from the input features, and is therefore computationally of low complexity. Finally, the NN input values are obtained from the current time frame only, which makes the approach fundamentally an online separation method, even though the SSL is here performed offline.

This paper is organized as follows. Section 2 reviews the signal model and beamforming. Spatial features used for training the neural network are presented in Section 3 . Section 4 describes the neural network model, the training process, and the proposed separation method. Section 5 describes the used recording arrangement. Section 6 describes the objective and perceptual performance of distant talker separation in contrast to a state-of-the art complex NMF approach, a spatial separation algorithm, and conventional beamforming approaches. Finally, Section 7 concludes the discussion.

\section{Signal Model and Beamforming}

In a reverberant multisource environment the signal $s_{k}(t)$ emitted by $k$ th sound source $(k=1, \ldots, K)$ at location $\mathbf{s}^{k} \in \mathbb{R}^{3}$ is captured with the $i$ th microphone $(i=1, \ldots, M)$ at location $\mathbf{m}_{i} \in \mathbb{R}^{3}$ as

$$
x_{i}(t)=\sum_{k=1}^{K} s_{k}(t) * h_{i}^{k}(t)+n_{i}(t),
$$

where $n_{i}(t)$ is i.i.d. sensor noise, and $h_{i}^{k}(t)$ is the impulse response between source $k$ and microphone $i$, and $t$ is time. In non-reverberant space, the impulse response consists only of the direct path component $h_{i}^{k}(t)=a_{i}^{k} \delta_{i}^{k}\left(t-\tau_{i}^{k}\right)$ where the propagation delay is $\tau_{i}^{k}=c^{-1}\left\|\mathbf{s}^{k}-\mathbf{m}_{i}\right\|$, attenuation is $a_{i}^{k} \propto\left\|\mathbf{s}^{k}-\mathbf{m}_{i}\right\|^{-1}$, and $\|\cdot\|$ denotes Euclidean norm with $c$ being speed of sound.

The continuous signals $x_{i}(t)$ are discretized and processed in the frequency domain using short-time Fourier transform (STFT) denoted as $X_{i}(\ell, n)$, where $n$ is time index of a processing window at discrete frequency index $0 \leq \ell \leq N_{\mathrm{DFT}}-1$. Let Cartesian vector $\boldsymbol{\xi}^{k}=\left[\xi_{x}^{k}, \xi_{y}^{k}, \xi_{z}^{k}\right]^{\mathrm{T}}$ with length $\left\|\boldsymbol{\xi}^{k}\right\|=c^{-1}$ denote $k$ th source emitted wavefront's direction of arrival (DOA) with respect to microphone array origin. The delay-and-sum beamformer (DSB) steered towards the $k$ th source with post-filtering aligns and sums the microphone signals and applies a realvalued gain $G(\ell, n)$

$$
Y^{k}(\ell, n)=\underbrace{\frac{1}{M} \sum_{i=1}^{M} X_{i}(\ell, n) \exp \left\{j \omega_{\ell} \cdot \tau_{i, 1}\left(\boldsymbol{\xi}^{k}\right)\right.}_{Y_{\mathrm{DSB}}^{k}(\ell, n)}\} \cdot G(\ell, n),
$$

where $\omega_{\ell}=2 \pi \ell / N_{\mathrm{DFT}}$ is radial frequency, and $Y_{\mathrm{DSB}}^{k}(\ell, n)$ is the DSB output without post-filtering. The steering delays are obtained from time difference of arrival (TDOA) values using $k$ th source DOA information as

$$
\tau_{i, j}\left(\boldsymbol{\xi}^{k}\right)=\left(\mathbf{m}_{i}-\mathbf{m}_{j}\right)^{\mathrm{T}} \boldsymbol{\xi}^{k} .
$$


Several online SSL methods in case of multiple sources in reverberant environments have been proposed by e.g. Valin et al. (2007); Brutti et al. (2009); Pertilä and Hämäläinen (2010); Fallon (2008). In the presented work any suitable SSL method for DOA estimation could be applied. However, the DOA estimation is not the focus of this work. Therefore, the well known technique of steered response power with phase transform weighting (SRP-PHAT) (DiBiase et al., 2001) is used to obtain a likelihood for a set of equally spread source candidate directions. The SRP-PHAT is aggregated over the mixture signal and weighted with the log-energy of each processing frame to account for silent periods, refer to (Pertilä and Nikunen, 2014) for details. Then, the $K$ largest peaks are chosen to represent the desired $K$ source directions, where $K$ is assumed known.

\section{Spatial Features}

Given the look direction $\boldsymbol{\xi}^{k}$, a spatial feature at time frame $n$ denoted as $u\left(\ell, n \mid \boldsymbol{\xi}^{k}\right)$ is calculated by averaging the differences between the measured $\left(\hat{\phi}_{i j}\right)$ and theoretical phase differences $\left(\phi_{i j}\right)$ over all $P$ unique microphone pairs $\{i, j\}$ (Pertilä and Nikunen, 2014)

$u\left(\ell, n \mid \boldsymbol{\xi}^{k}\right)=\frac{1}{P} \sum_{\forall\{i, j\}} \cos (\underbrace{\angle X_{i}(\ell, n)-\angle X_{j}(\ell, n)}_{\hat{\phi}_{i j}}-\underbrace{\omega_{\ell} \cdot \tau_{i, j}\left(\boldsymbol{\xi}^{k}\right)}_{\phi_{i j}})$,

where $\angle Z$ denotes phase of complex number $Z$. The $\cos (\cdot)$ function is used to handle the $2 \pi$ ambiguity by wrapping the phase values into the range $[-1,+1]$. The feature is a scalar value bounded in range $[-1,+1]$, where value $u\left(\ell, n \mid \boldsymbol{\xi}^{k}\right)=1$ indicates perfect agreement between measured and theoretical phase differences between all microphone pairs.

The information about signals arriving from non-look directions is taken from the average value over non-look direction feature values

$$
v\left(\ell, n \mid \boldsymbol{\xi}^{k}\right)=\frac{1}{L} \sum_{l=1}^{L} u\left(\ell, n \mid \boldsymbol{\xi}_{l}^{k}\right),
$$

where direction vectors $\boldsymbol{\xi}_{l}^{k}, \ldots, \boldsymbol{\xi}_{L}^{k}$ are spread around the DOA space, and do not contain angles close to the desired looking direction $\boldsymbol{\xi}^{k}$.

\section{Neural network for T-F mask prediction}

The NN input vector $\mathbf{f}^{k}(n)$ at time frame $n$ consists of phase based features towards a source (4) and features towards other directions (5) for all frequency bins corresponding to real part of the spectrum $\ell=\left[0, \ldots, N_{\mathrm{DFT}} / 2-\right.$ $1]$

$$
\begin{aligned}
f_{2 \ell}^{k}(n) & =u\left(\ell, n \mid \boldsymbol{\xi}^{k}\right), \\
f_{2 \ell+1}^{k}(n) & =v\left(\ell, n \mid \boldsymbol{\xi}^{k}\right) .
\end{aligned}
$$

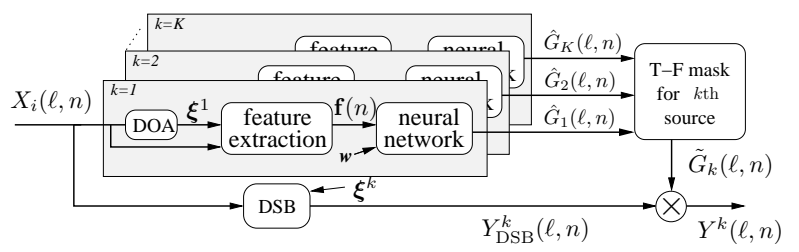

Figure 1: Using the trained neural network with weights $\mathbf{w}$ for separating $k$ th source at direction $\boldsymbol{\xi}^{k}$ from noisy microphone array signals $X_{i}(\ell, n), i=1, \ldots, M$.

The size of the input feature is therefore $\mathbf{f}^{k}(n) \in \mathbb{R}^{N_{\mathrm{DFT}}}$.

A NN with one hidden layer, often referred as the multilayer perceptron (MLP), with twice the nodes in the hidden layer compared to the input layer is used. This structure was selected after examining the output error when adding more hidden layers and increasing the width of each hidden layer. However, only widening of the single hidden layer was found to significantly reduce the error, unlike adding more hidden layers. The (predicted) T-F mask value of the $\ell$ th frequency is the corresponding value of the $\ell$ th output layer node at time frame $n$ (Bishop, 2006)

$$
\hat{G}_{k}(\ell, n)=\sigma\left(\sum_{j=1}^{2 N_{\mathrm{DFT}}} w_{\ell j}^{(2)} \sigma\left(\sum_{i=1}^{N_{\mathrm{DFT}}} w_{j i}^{(1)} f_{i}^{k}(n)+w_{j 0}^{(1)}\right)+w_{\ell 0}^{(2)}\right)
$$

where $w_{j i}^{(1)}$ denotes the weight given for the $i$ th input by hidden layer neuron $j, w_{\ell j}^{(2)}$ is the weight given for the output of the $j$ th hidden layer neuron by the $\ell$ th node of the output layer, $\sigma(\cdot)$ is the logistic sigmoid function, $w_{j 0}^{(1)}$ and $w_{\ell 0}^{(2)}$ are bias weights. Note that the logistic function is also applied at the output nodes, which bounds the T-F mask value between the desired range $[0,1]$.

\subsection{Processing of predicted T-F masks for separation}

The NN (8) predicts the T-F masks independently for each source. A soft valued post-processed T-F mask is constructed using all predicted source masks to exploit the information between competing sources

$$
\tilde{G}_{k}(\ell, n)=\left\{\begin{array}{cc}
\hat{G}_{k}(\ell, n), & \text { if } \hat{G}_{k}(\ell, n)-\hat{G}_{k^{\prime}}(\ell, n) \geq L C \forall k^{\prime} \neq k \\
0, & \text { otherwise }
\end{array} .\right.
$$

This preserves the soft mask value generated by the neural network while promoting mask sparsity, which is controlled by $L C$. When $L C=-1$ each source is separated by applying directly the predicted mask, and when $L C=0$ each T-F mask value is assigned to a single target only. With $L C>0$, mask values that do not exceed every other mask value by a margin $L C$ are set to zero. The procedure is analogous to the ideal binary mask (IBM), where the selection parameter is SNR and a binary mask value is obtained instead of a real-valued one (Wang, 2008). 


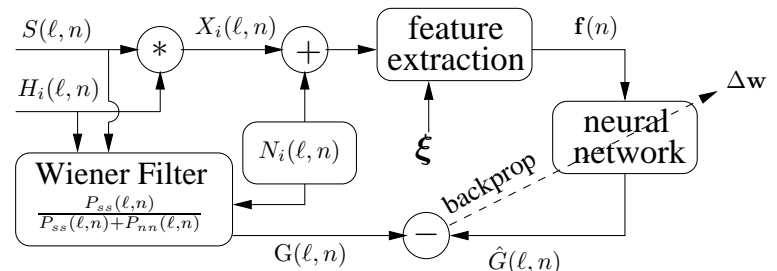

Figure 2: Training of the neural network is illustrated. Features are generated from simulated microphone signals using clean speech $S(\ell, n)$ convolved with simulated room impulse responses $H_{i}(\ell, n)$ from a source at direction $\boldsymbol{\xi}$ with added noise $N_{i}(\ell, n)$. The target values $G(\ell, n)$ are obtained using the Wiener filter. The backpropagation algorithm changes the weights by $\Delta \mathbf{w}$ based on the prediction error, where $\mathbf{w}$ denotes the set of all weights.

\subsection{Algorithm Description}

The complete algorithm for separation of the $k$ th source at time frame $n$ is summarized as Alg. 1. Refer to Fig. 1 for a block diagram of separating the $k$ th source.

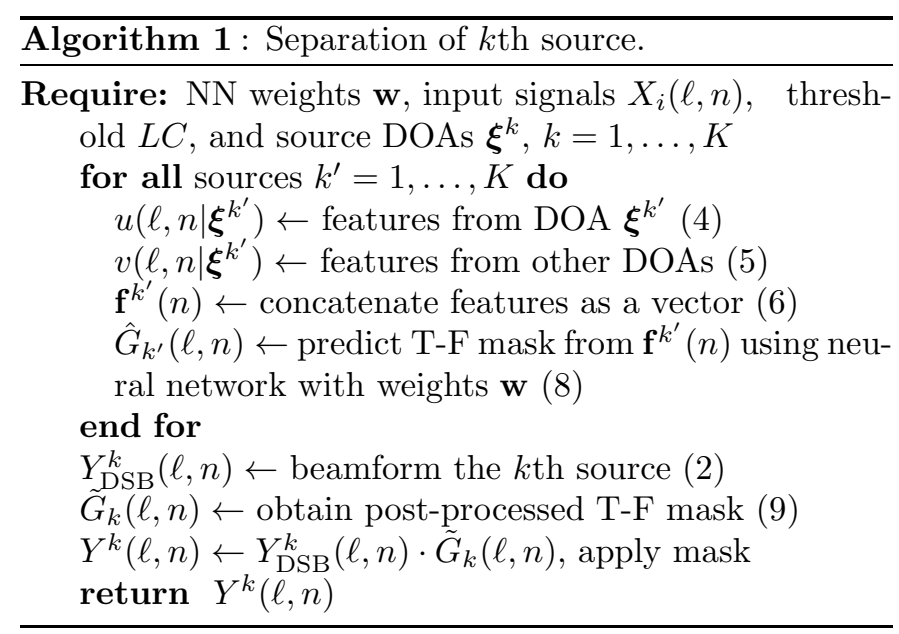

\subsection{Simulation Data for Training}

The network training is done using simulations to generate realistic speech data in typical rooms. The image method by Allen and Berkley (1979) is used to simulate room impulse responses between different source positions and microphones arranged in a circular array with $10 \mathrm{~cm}$ radius in the middle of the room. Two different rooms with reverberation time $T_{60}$ values $300 \mathrm{~ms}$ and $600 \mathrm{~ms}$ were simulated. In both rooms the source was placed at two separate distances of $1.2 \mathrm{~m}$ and $2.1 \mathrm{~m}$ from the array towards a random azimuth direction. White Gaussian i.i.d. noise was added into the input signal to further diversify the conditions from which the data is obtained. The SNR at the microphones was drawn from a uniform distribution in range $[12,36] \mathrm{dB}$.
The target values for the T-F mask $G(\ell, n)$ are obtained using the Wiener filter (Diethorn, 2004)

$$
G(\ell, n)=\frac{P_{s s}(\ell, n)}{P_{s s}(\ell, n)+P_{n n}(\ell, n)},
$$

where $P_{s s}(\ell, n)$ and $P_{n n}(\ell, n)$ are the signal and noise power spectrums. The signal power spectrum $P_{s s}(\ell, n)$ contains only the direct path component of the 1st channel's impulse response (i.e. $h_{1}^{k}(t)=a_{1}^{k} \delta\left(t-\tau_{1}^{k}\right)$ ) convolved with the signal $s_{k}(t)$. In the enhancement framework of Pertilä and Nikunen (2014) the noise power spectrum $P_{n n}(\ell, n)$ contains only the added noise $n_{i}(t)$. Here, also the early and late reverberation components of the target speaker are considered as noise. This de-reverberates the output signal by reducing T-F values of non-target directions. Consequently, potential interfering sources in nontarget direction are attenuated. The network is therefore taught to remove source reverberation and preserve only the direct path information, i.e., mimic the output of a close-talk microphone.

100 sentences from the TIMIT database (Garofolo et al., 1993) were captured in both rooms for both distances, resulting in 400 sentences. The sampling rate was $16 \mathrm{kHz}$, and 512 sample Hann windows were used with $75 \%$ overlap between adjacent windows. $L=25$ non-source directions were used in (5). The error backpropagation algorithm 11 was used to train the network (8) using stochastic gradient descent with learning rate $\mu=0.1$. The data was divided into $90 \%$ training, $5 \%$ testing, and $5 \%$ for evaluation. The backpropagation was run on the training data until the error on the testing data reached a minimum, which did not decrease during five successive iterations. After 18 iterations, the final set of weights was obtained. The weights resulted in low mean square error of 0.007 with the unseen evaluation set indicating a success of the training. See Fig. 2 for a block diagram representation of generation the training data and the training process.

\section{Recording Descriptions}

The evaluation material was recorded in two different rooms. The first being an audio laboratory (Room 1) with floor dimensions $4.0 \times 4.5 \mathrm{~m}$ and low reverberation time $\mathrm{T}_{60}=260 \mathrm{~ms}$ with critical distance $r_{c}=1.0 \mathrm{~m}$ and the second being a regular meeting room (Room 2) with floor dimensions $5.1 \times 6.6 \mathrm{~m}$ and moderate reverberation time $\mathrm{T}_{60}=370 \mathrm{~ms}$ with $r_{c}=1.2 \mathrm{~m}$. The critical distance (Kuttruff, 2009) at which the sound energy of the reverberation is equal to that of the direct path is approximated as $r_{c} \approx 0.056 \sqrt{g V / T_{60}}$, where $V$ is room volume, and $g=1.62$ is the directivity factor modeled by using the average human speech directivity index of $2.1 \mathrm{~dB}$ measured by Monson et al. (2012). The recording locations

${ }^{1}$ Matlab v.2013b with the Deep Learn Toolbox was used, http://github.com/rasmusbergpalm/DeepLearnToolbox. 


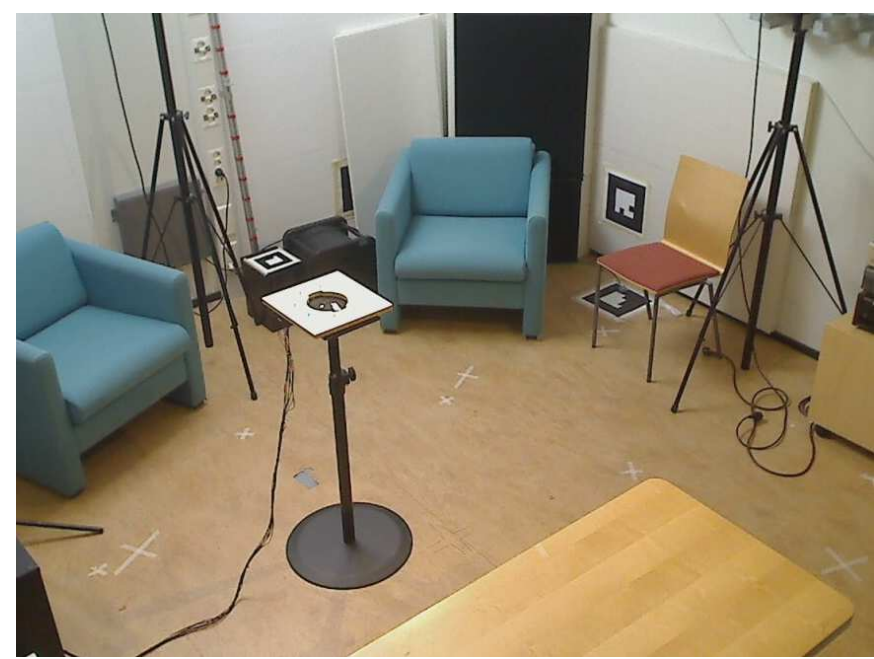

(a) Room 1: floor area is $4.0 \times 4.5 \mathrm{~m}$ with reverberation time $\mathrm{T}_{60} 260 \mathrm{~ms}$.

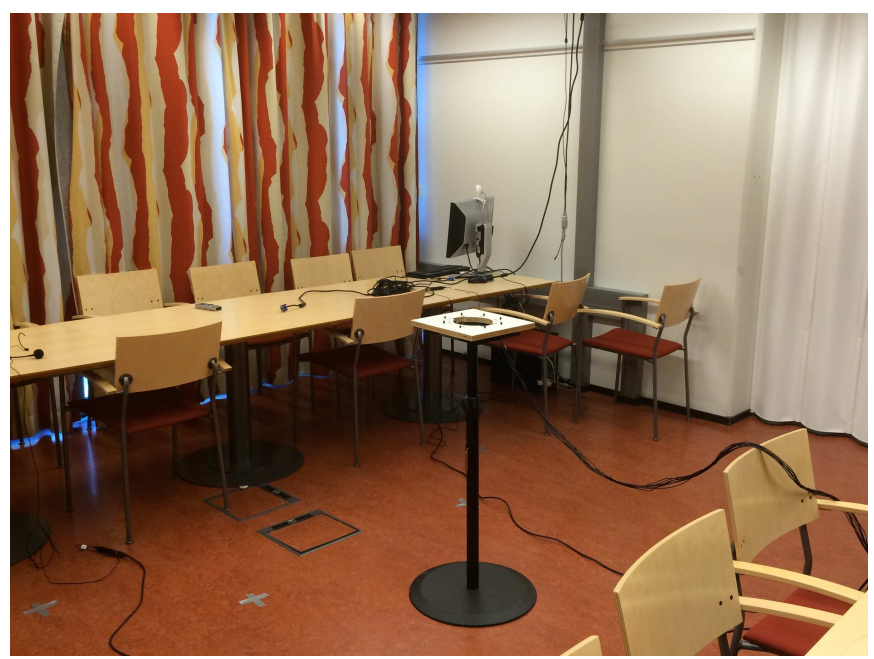

(b) Room 2: floor area is $5.1 \times 6.6 \mathrm{~m}$ with reverberation time $\mathrm{T}_{60} 370 \mathrm{~ms}$.

Figure 3: Recording setup of both rooms is shown. The used circular array is mounted on a speaker stand.

are referred as "Room 1" and "Room 2" in the result tables. An eight-channel circular microphone array with $10 \mathrm{~cm}$ radius was placed on a stand at $1.0 \mathrm{~m}$ height and the array was approximately centered inside the rooms. Recording equipment was present inside the rooms and both of the recording locations had air conditioning on. The Room 2 had an overall higher background noise due to the louder air conditioning. Sennheiser MKE 2 electret condenser microphones were used in the array. Headworn close-talk condenser microphones (AKG C-520) were used to capture the reference signal. The data was sampled at $48 \mathrm{kHz}$ using RME octamic II and RME UFX. The recording setup of both rooms is depicted in Fig. 3.

The phonetically balanced Harvard sentences (Rothauser et al., 1969) were read by four different male speakers standing still and facing the array. Speech from two distances of $110 \mathrm{~cm}$ (near) and $170 \mathrm{~cm}$ (far) between the array center and speaker head position were recorded. The angle between adjacent speakers was $90^{\circ}$. Refer to Fig. 4 for an illustration of the setup. The source positioning and overall recording setting was identical in both rooms.

The data was segmented into sentences, and the corresponding microphone signals were normalized using their RMS values. Synthetic mixtures of overlapping speakers were then generated by mixing two and three sources together. Three separate sentences from each person were mixed in all possible pairwise permutations to generate 54 two-speaker mixtures. For the three source case, all speaker permutations using two separate sentences from each speaker were generated to produce 32 three-speaker mixtures.

\section{Separation Results}

The audio was downsampled to $16 \mathrm{kHz}$ before processing except for the CNMF, which uses $24 \mathrm{kHz}$ sampling rate. The same processing parameters (window length, overlap) as in the generation of the training data was applied, refer to Section 4.3

\subsection{Separation Performance Evaluation}

Three metrics are used to evaluate the objective performance related to the efficiency of the separation. The first metric is the frequency-weighted $S N 2^{2}$ (fwSNRseg), which is the weighted average of SNR values of each frequency band ( $\mathrm{Hu}$ and Loizou, 2008). The fwSNRSeg has been reported to be correlated $(\rho=0.84)$ with subjective listening ratings of speech quality by $\mathrm{Hu}$ and Loizou (2008). The other objective separation metrics are source to interference ratio (SIR) and source to distortion ratio (SDR). The latter metrics are obtained from the blind source separation toolkit3 v3.0. The SIR expresses the amount of target signal present in the separated signal divided by the amount of interfering sources. The SDR expresses to amount of target signal present in the separated signal divided by the interfering sources and artifact, refer to (Vincent et al., 2006).

The intelligibility of the separation results was analyzed with the short-time objective intelligibility measure (STOI) (Taal et al., 2011), which is designed for predicting the intelligibility of T-F weighted noisy speech.

\footnotetext{
${ }^{2} \mathrm{fwSNR}$ seg is implemented using 25 MEL-frequency bands, RMS normalized signals, and weight parameter $\gamma=0.2$.

${ }^{3}$ http://bass-db.gforge.inria.fr/bss_eval/

${ }^{4}$ http://www. ceestaal.nl/stoi.zip
} 


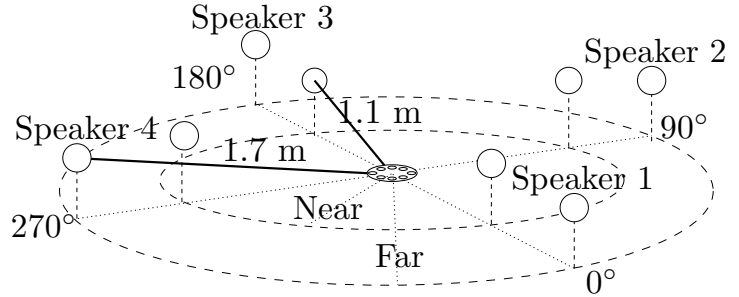

Figure 4: Four speakers at $90^{\circ}$ spacing at two separate distances from the eight-channel circular array (radius $10 \mathrm{~cm}$ ) are illustrated as circles. The array is at $1.0 \mathrm{~m}$ height and speaker height is approximately $1.8 \mathrm{~m}$. The resulting speaker head distance from array center is $1.1 \mathrm{~m}$ in the "Near" case, and $1.7 \mathrm{~m}$ in the "Far" case.

\subsection{Contrast methods}

The proposed method's (Alg. 1) post-filter threshold (9) was set empirically to $L C=-0.15$ using a subset of recordings by preferring a tradeoff between high STOI and $\mathrm{SIR}$ values. Its output is contrasted between the scores obtained from i) an unprocessed microphone signal (Mic.), ii) the output of the DSB, iii) the output of the MVDR beamformer, iv) CNMF by Nikunen and Virtanen (2014b), and v) multiple target tracking and spatial source separation algorithm (MTT-SEP) by Pertilä (2013). Note that the methods i)-iii) are not separation methods unlike the other methods.

The contrast method iii) MVDR beamformer used diagonal loading and the estimation of the required noise covariance matrix was implemented using the sample covariance method, i.e. forming covariance matrices of each frequency from past time frames, cf. Tashev (2009). The number of time frames and the diagonal loading value were empirically determined to optimize the separation metrics for a subset of recordings. MVDR is assumed to capture the combination of stationary noise statistics while also providing attenuation of the non-stationary competing sources and reverberation.

The contrast method iv) is based on CNMF, which utilizes spectral redundancy of sources and their spatial cues in estimating the separation parameters. The algorithm operates in the covariance domain and the parameters to be estimated are the source magnitude spectrogram and its mixing in form of covariance matrix for each T-F point. The method uses only four microphones and was run for 1000 iterations with other parameters specified in (Nikunen and Virtanen, 2014b). The scores were calculated by downsampling the separated signals to $16 \mathrm{kHz}$. Nikunen and Virtanen (2014b) compared the method to conventional frequency domain ICA separation by Sawada et al. (2007) and another CNMF based separation proposed by Sawada et al. (2011), thus allowing indirect comparison of the proposed method against conventional approaches.

The contrast method v) MTT-SEP by Pertilä (2013) is based on multi-target tracking (MTT) and T-F masking. The actual T-F mask is obtained by dividing the spatial likelihood a desired target with the likelihood accumulated from all other directions in each frequency bands of each frame. The approach is engineered for objective separation performance and can produce musical noise artifacts.

\subsection{Separation Results}

The upper half of Table 1 presents results averaged over 54 mixtures of two sources (total of 108 separated sentences) for both the near and the far source distances in the low reverberant Room 1 . The fwSNRSeg and STOI are highest for the proposed method for both distances. The separation performance in terms of SIR is clearly highest for MTT-SEP, while SDR scores are within $1.6 \mathrm{~dB}$ range between MVDR, CNMF, MTT-SEP and the proposed method. Increasing the source distance from $1.1 \mathrm{~m}$ (near) to $1.7 \mathrm{~m}$ (far) decreases the overall scores for each method. The lower half of Table 1 presents results for three concurrent speakers in Room 1. The overall scores are lower compared to the two source case due to increased difficulty of the task. The proposed method again has the highest fwSNRseg and STOI scores and the MTTSEP again has the highest score for the SIR metric, while MVDR, CNMF, and MTT-SEP produce similar SDR values.

The upper half of Table 2 presents results for the moderately reverberant Room 2. The proposed method's intelligibility score (STOI) is highest for near and far case for both two and three sources. Similarly the proposed method has the highest frequency-weighted SNR. The MTT-SEP method has best performance in terms of SIR, and SDR, which are objective metrics that do not considerer perceptual aspects.

Since the CNMF output includes reverberation, unlike the outputs of other separation methods, it's performance is subpar for estimating the close-talk signal. This is indicated by the low fwSNRseg score.

\subsection{Summary of Results}

Considering both rooms, both distances and the number of simultaneous sources the proposed algorithm achieved statistically significant highest average fwSNRseg values (all pairwise $p$-values $\ll 0.01$ ). Similarly, the proposed method achieved statistically significant highest average intelligibility scores (STOI) ( $p$-values $\ll 0.01)$.

In contrast, the MTT-SEP has the highest separation score in terms of SIR in all scenarios. The SDR is also highest for the MTT-SEP in the more reverberant room, while in the low reverberant room the SDR scores are high for MVDR, CNMF, and MTT-SEP. Informal listening experience suggests that the proposed method has a smaller amount of artifacts compared to MTT-SEP, while still being able to separate the source signals. This observation is inline with the obtained scores. Furthermore, while the MTT-SEP method has high objective separation scores 
Table 1: Average separation performance in low reverberant Room 1 with two (upper part of table) and three (lower part of table) simultaneous speakers $(K=2, K=3)$. Results for speaker distances Near $(1.1 \mathrm{~m})$ and Far $(1.7 \mathrm{~m})$ from the array center are given.

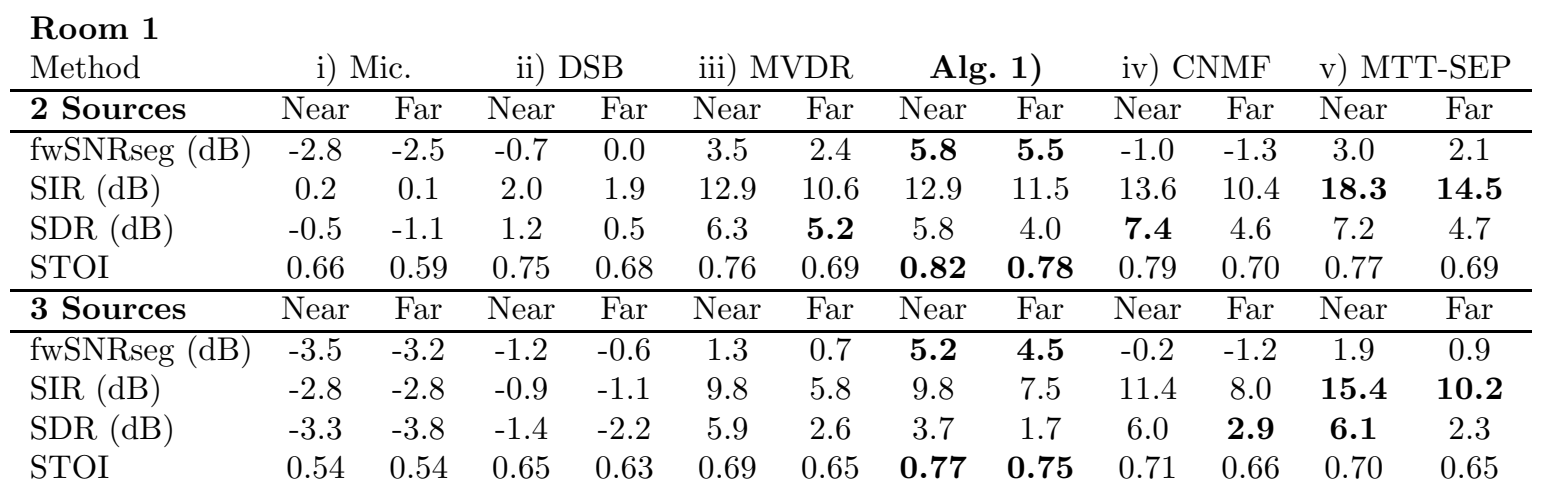

Table 2: Average separation performance in moderately reverberant Room 2 with two (upper part of table) and three (lower part of table) simultaneous speakers $(K=2, K=3)$. Results for speaker distances Near $(1.1 \mathrm{~m})$ and Far $(1.7 \mathrm{~m})$ from array center are given.

\begin{tabular}{lcccccccccccc}
$\begin{array}{l}\text { Room 2 } \\
\text { Method }\end{array}$ & \multicolumn{1}{c}{ i) } & Mic. & \multicolumn{1}{c}{ ii) } & DSB & iii) MVDR & \multicolumn{2}{c}{ Alg. 1) } & \multicolumn{1}{c}{ iv) CNMF } & \multicolumn{2}{c}{ v) MTT-SEP } \\
\hline 2 Sources & Near & Far & Near & Far & Near & Far & Near & Far & Near & Far & Near & Far \\
\hline fwSNRseg (dB) & -2.7 & -2.4 & -0.9 & -0.4 & 3.5 & 2.5 & $\mathbf{4 . 9}$ & $\mathbf{4 . 3}$ & -0.9 & -1.3 & 2.3 & 2.3 \\
SIR (dB) & 0.2 & 0.1 & 2.2 & 2.3 & 13.0 & 12.8 & 12.6 & 12.6 & 12.7 & 12.5 & $\mathbf{1 8 . 5}$ & $\mathbf{1 7 . 1}$ \\
SDR (dB) & -1.5 & -2.5 & -0.1 & -1.2 & 5.0 & 4.2 & 3.6 & 2.4 & 4.6 & 3.1 & $\mathbf{6 . 2}$ & $\mathbf{4 . 7}$ \\
STOI & 0.66 & 0.60 & 0.75 & 0.69 & 0.77 & 0.70 & $\mathbf{0 . 8 2}$ & $\mathbf{0 . 7 9}$ & 0.78 & 0.72 & 0.77 & 0.71 \\
\hline 3 Sources & Near & Far & Near & Far & Near & Far & Near & Far & Near & Far & Near & Far \\
\hline fwSNRseg (dB) & -3.4 & -3.2 & -1.4 & -1.1 & 1.3 & 0.6 & $\mathbf{4 . 4}$ & $\mathbf{3 . 1}$ & -0.1 & -0.7 & 1.7 & 1.4 \\
SIR (dB) & -2.8 & -2.9 & -0.9 & -0.8 & 8.0 & 7.3 & 7.9 & 7.6 & 10.4 & 10.0 & $\mathbf{1 4 . 4}$ & $\mathbf{1 2 . 7}$ \\
SDR (dB) & -4.1 & -4.9 & -2.5 & -3.4 & 3.4 & 2.2 & 1.1 & -0.1 & 3.6 & 2.3 & $\mathbf{4 . 5}$ & $\mathbf{2 . 6}$ \\
STOI & 0.57 & 0.53 & 0.69 & 0.62 & 0.71 & 0.64 & $\mathbf{0 . 7 7}$ & $\mathbf{0 . 7 4}$ & 0.72 & 0.67 & 0.71 & 0.65
\end{tabular}

(SIR, SDR), metrics related to the perceptual quality (fwSNRseg) and intelligibility (STOI) are somewhat sacrificed to achieve this result.

\section{Conclusions}

This work presents a speech separation method based on predicting a T-F mask with a feed-forward neural network (NN) utilizing features obtained from microphone array's phase measurements. The features are independent of absolute source angle and therefore allow the use of a single NN for any number of sources in any directions. The NN is trained using real speech samples in different simulated reverberant conditions. The T-F masks are post-processed to exploit information between sources. The masks are then in turn applied to the beamformed signal to separate each of the desired sources. The method was tested with distant talker speech mixtures from array recordings in two separate rooms with two speaker distances. The method provided higher instrumental measure for intelligibility and frequency weighted SNR compared two stateof-the-art separation approaches and conventional beamforming approaches.
Samples from the proposed method are available online at http://www.cs.tut.fi/ pertila/nnsep/.

\section{Acknowledgments}

The corresponding author wishes to thank Finnish Academy project no. 138803 for its role in funding the research.

\section{References}

Allen, J., Berkley, D., 1979. Image Method for Efficiently Simulating Small-Room Acoustics. J. Acoust. Soc. Am. 65 (4), 943 - 950.

Ayllon, D., Gil-Pita, R., Rosa-Zurera, M., 2013. Rate-Constrained Source Separation for Speech Enhancement in WirelessCommunicated Binaural Hearing Aids. EURASIP Journal on Advances in Signal Processing 2013 (1).

Bishop, C., 2006. Pattern Recognition and Machine Learning. Information Science and Statistics. Springer.

Brutti, A., Omologo, M., Svaizer, P., 2009. A Sequential Monte Carlo Approach for Tracking of Overlapping Acoustic Sources. In: Proc. 17th European Signal Processing Conference (EUSIPCO’09). pp. 2559-2563.

DiBiase, J., Silverman, H., Brandstein, M., 2001. Robust Localization in Reverberant Rooms. In: Brandstein, M., Ward, D. (Eds.), Microphone Arrays. Springer-Verlag, Ch. 8, pp. $157-180$. 
Diethorn, E., 2004. Subband Noise Reduction Methods for Speech Enhancement. In: Huang, Y., Benesty, J. (Eds.), Audio Signal Processing for Next-Generation Multimedia Communication Systems. Kluwer Academic Publishers, Ch. 4, pp. 91-115.

Fallon, M., May 2008. Multi target acoustic source tracking with an unknown and time varying number of targets. In: Hands-Free Speech Communication and Microphone Arrays, 2008. HSCMA 2008. pp. $77-80$

Fastl, H., Zwicker, E., 1990. Psychoacoustics - facts and models. In: Huang, T. S., Schroeder, M. R., Kohonen, T. (Eds.), Springer Handbook of Acoustics, 3rd Edition. Springer, pp. 429-457.

Garofolo, J. S., Lamel, L. F., Fisher, W. M., Fiscus, J. G., Pallett, D. S., Dahlgren, N. L., Zue, V., 1993. TIMIT Acoustic-Phonetic Continuous Speech Corpus. Linguistic Data Consortium, Philadelphia.

Gemmeke, J. F., Virtanen, T., Hurmalainen, A., 2011. ExemplarBased Sparse Representations for Noise Robust Automatic Speech Recognition. IEEE Transactions on Audio, Speech, and Language Processing 19 (7), 2067-2080.

Healy, E. W., Yoho, S. E., Wang, Y., Wang, D., 2013. An Algorithm to Improve Speech Recognition in Noise for Hearing-Impaired Listeners. The Journal of the Acoustical Society of America 134 (4), 3029-3038.

Hu, Y., Loizou, P., Jan 2008. Evaluation of Objective Quality Measures for Speech Enhancement. IEEE Transactions on Audio, Speech, and Language Processing 16 (1), 229-238.

Hummersone, C., Stokes, T., Brookes, T., 2014. On the Ideal Ratio Mask as the Goal of Computational Auditory Scene Analysis. In: Naik, G. R., Wang, W. (Eds.), Blind Source Separation: Advances in Theory, Algorithms and Applications. Springer, Ch. 12.

Hyvärinen, A., Oja, E., 2000. Independent component analysis: algorithms and applications. Neural Networks 13 (4-5), $411-430$.

Jiang, Y., Wang, D., Liu, R., 2014. Binaural Deep Neural Network Classification for Reverberant Speech Segregation. In: Proc. 15th Annual Conference of the International Speech Communication Association (Interspeech).

Kim, G., Lu, Y., Hu, Y., Loizou, P. C., 2009. An Algorithm that Improves Speech Intelligibility in Noise for Normal-Hearing Listeners. The Journal of the Acoustical Society of America 126 (3), 1486-?1494.

Koning, R., Madhu, N., Wouters, J., 2014. Ideal Time-Frequency Masking Algorithms Lead to Different Speech Intelligibility and Quality in Normal-Hearing and Cochlear Implant Listeners. IEEE Transactions on Biomedical Engineering(accepted for publication.

Kuttruff, H., 2009. Room Acoustics, 5th Edition. Spon Press.

Lefkimmiatis, S., Maragos, P., 2007. A Generalized Estimation Approach for Linear and Nonlinear Microphone Array Post-Filters. Speech communication 49 (7), 657-666.

Maas, A. L., Le, Q. V., O'Neil, T. M., Vinyals, O., Nguyen, P., Ng, A. Y., 2012. Recurrent Neural Networks for Noise Reduction in Robust ASR. In: Proc. 13th Annual Conference of the International Speech Communication Association (Interspeech).

Madhu, N., Spriet, A., Jansen, S., Koning, R., Wouters, J., Jan 2013. The Potential for Speech Intelligibility Improvement Using the Ideal Binary Mask and the Ideal Wiener Filter in Single Channel Noise Reduction Systems: Application to Auditory Prostheses. IEEE Transactions on Audio, Speech, and Language Processing 21 (1), 63-72.

McCowan, I., Bourlard, H., Nov 2003. Microphone Array Post-Filter based on Noise Field Coherence. IEEE Transactions on Speech and Audio Processing 11 (6), 709-716.

Mohammadiha, N., Smaragdis, P., Leijon, A., 2013. Supervised and Unsupervised Speech Enhancement Using Nonnegative Matrix Factorization. IEEE Transactions on Audio, Speech, and Language Processing 21 (10), 2140-2151.

Monson, B. B., Hunter, E. J., Story, B. H., 2012. Horizontal Directivity of Low- and High-Frequency Energy in Speech and Singing. The Journal of the Acoustical Society of America 132 (1), 433441.

Narayanan, A., Wang, D., 2013. Ideal Ratio Mask Estimation Using Deep Neural Networks for Robust Speech Recognition. In: Proc.
IEEE International Conference on Acoustics, Speech and Signal Processing (ICASSP).

Nikunen, J., Virtanen, T., 2014a. Direction of Arrival Based Spatial Covariance Model for Blind Sound Source Separation. IEEE Transactions on Audio, Speech, and Language Processing 22 (4), $727-739$

Nikunen, J., Virtanen, T., 2014b. Multichannel Audio Separation by Direction of Arrival Based Spatial Covariance Model and Non-negative Matrix Factorization. In: Proceedings of International Conference on Acoustics, Speech, and Signal Processing (ICASSP'14). pp. 6727-6731.

Ozerov, A., Fevotte, C., 2010. Multichannel Nonnegative Matrix Factorization in Convolutive Mixtures for Audio Source Separation. IEEE Transactions on Audio, Speech, and Language Processing 18 (3), 550-563.

Pertilä, P., 2013. Online Blind Speech Separation Using Multiple Acoustic Speaker Tracking and Time-Frequency Masking. Computer Speech \& Language 27 (3), 683 - 702.

Pertilä, P., Hämäläinen, M. S., 2010. A Track Before Detect Approach for Sequential Bayesian Tracking of Multiple Speech Sources. In: Proc. IEEE International Conference on Acoustics, Speech, and Signal Processing (ICASSP'10).

Pertilä, P., Nikunen, J., 2014. Microphone Array Post-Filtering Using Supervised Machine Learning for Speech Enhancement. In: Proc. 15th Annual Conference of the International Speech Communication Association (Interspeech).

Raj, B., Virtanen, T., Chaudhuri, S., Singh, R., 2010. Non-Negative Matrix Factorization Based Compensation of Music for Automatic Speech Recognition. In: Proc. 11th Annual Conference of the International Speech Communication Association (Interspeech). pp. $717-720$.

Roman, N., Wang, D., Brown, G. J., 2003. Speech Segregation based on Sound Localization. J. Acoust. Soc. Am. 114 (4), 2236-2252.

Rothauser, E. H., Chapman, W. D., Guttman, N., Hecker, M. H. L., Nordby, K. S., Silbiger, H. R., Urbanek, G. E., Weinstock, M., Sep 1969. IEEE Recommnded Pratice for Speech Quality Measurements. Audio and Electroacoustics, IEEE Transactions on 17 (3), $225-246$.

Sawada, H., Araki, S., Mukai, R., Makino, S., 2007. Grouping Separated Frequency Components by Estimating Propagation Model Parameters in Frequency-Domain Blind Source Separation. IEEE Transactions on Audio, Speech, and Language Processing 15 (5), 1592-1604

Sawada, H., Kameoka, H., Araki, S., Ueda, N., 2011. New Formulations and Efficient Algorithms for Multichannel NMF. In: IEEE Workshop on Applications of Signal Processing to Audio and Acoustics (WASPAA). IEEE, pp. 153-156.

Sawada, H., Kameoka, H., Araki, S., Ueda, N., 2013. Multichannel Extensions of Non-negative Matrix Factorization with Complexvalued Data. IEEE Transactions on Audio, Speech, and Language Processing 21 (5), 971-982.

Sawada, H., Mukai, R., Araki, S., Makino, S., 2004. A Robust and Precise Method for Solving the Permutation Problem of Frequency-Domain Blind Source Separation. IEEE Transactions on Speech and Audio Processing 12 (5), 530-538.

Schuller, B., Weninger, F., Wollmer, M., Sun, Y., Rigoll, G., 2010. Non-Negative Matrix Factorization as Noise-Robust Feature Extractor for Speech Recognition. In: Acoustics Speech and Signal Processing (ICASSP), 2010 IEEE International Conference on. IEEE, pp. 4562-4565.

Seltzer, M., Tashev, I., Acero, A., 2007. Microphone Array PostFilter using Incremental Bayes Learning to Track the Spatial Distributions of Speech and Noise. In: Proc. IEEE International Conference on Acoustics, Speech and Signal Processing (ICASSP).

Seltzer, M. L., Tashev, I., 2008. A Log-MMSE Adaptive Beamformer Using a Nonlinear Spatial Filter. In: Proc. 11th Int. Workshop on Acoustic Echo and Noise Control (IWAENC).

Simmer, K. U., Bitzer, J., Marro, C., 2001. Post-filtering techniques. In: Brandstein, M., Ward, D. (Eds.), Microphone Arrays. Springer, pp. 39-60.

Smaragdis, P., 1998. Blind Separation of Convolved Mixtures in the 
Frequency Domain. Neurocomputing 22 (1), 21-34.

Srinivasan, S., Roman, N., Wang, D., 2006. Binary and Ratio TimeFrequency Masks for Robust Speech Recognition. Speech Communication 48 (11), 1486-1501.

Swietojanski, P., Ghoshal, A., Renals, S., 2013. Hybrid Acoustic Models for Distant and Multichannel Large Vocabulary Speech Recognition. In: IEEE Workshop on Automatic Speech Recognition and Understanding (ASRU). IEEE, pp. 285-290.

Taal, C. H., Hendriks, R. C., Heusdens, R., Jensen, J., 2011. An Algorithm for Intelligibility Prediction of Time-Frequency Weighted Noisy Speech. IEEE Transactions on Audio, Speech, and Language Processing 19 (7), 2125-2136.

Tashev, I., Acero, A., 2006. Microphone Array Post-Processor Using Instantaneous Direction of Arrival. Proc. Int. Workshop on Acoustic Echo and Noise Control (IWAENC).

Tashev, I. J., 2009. Sound Capture and Processing: Practical Approaches. John Wiley \& Sons Ltd.

Valin, J., Michaud, F., Rouat, J., 2007. Robust Localization and Tracking of Simultaneous Moving Sound Sources Using Beamforming and Particle Filtering. Robotics and Autonomous Systems Journal (Elsevier) 55 (3), $216-228$.

Vincent, E., Gribonval, R., Fevotte, C., july 2006. Performance Measurement in Blind Audio Source Separation. IEEE Transactions on Audio, Speech, and Language Processing 14 (4), 1462 -1469.

Wang, D., 2005. On Ideal Binary Mask As the Computational Goal of Auditory Scene Analysis. In: Divenyi, P. (Ed.), SPEECH SEPARATION BY HUMANS AND MACHINES. Kluwer Academic Publishers, Ch. 12.

Wang, D., 2008. Time-Frequency Masking for Speech Separation and its Potential for Hearing Aid Design. Trends in Amplification 12, 332-353.

Wang, Y., Wang, D., July 2013. Towards Scaling Up ClassificationBased Speech Separation. IEEE Transactions on Audio, Speech, and Language Processing 21 (7), 1381-1390.

Weninger, F., Eyben, F., Schuller, B., 2014. Single-Channel Speech Separation With Memory-Enhanced Recurrent Neural Networks. In: Proc. IEEE International Conference on Acoustics, Speech, and Signal Processing (ICASSP).

Williamson, D. S., Wang, Y., Wang, D., 2014. A two-stage approach for improving the perceptual quality of separated speech. In: Proc. IEEE International Conference on Acoustics, Speech, and Signal Processing (ICASSP).

Woodruff, J., Wang, D., 2013. Binaural Detection, Localization, and Segregation in Reverberant Environments based on Joint Pitch and Azimuth Cues. IEEE Transactions on Audio, Speech, and Language Processing 21 (4), 806-815.

Ylmaz, O., Rickard, S., July 2004. Blind Separation of Speech Mixtures via Time-Frequency Masking. IEEE Transactions on Signal Processing 7 (52), 1830-1847.

Zelinski, R., 1988. A Microphone Array with Adaptive Post-Filtering for Noise Reduction in Reverberant Rooms. In: Proc. IEEE International Conference on Acoustics, Speech, and Signal Processing (ICASSP). 